\title{
App Gene Dosage Modulates Endosomal Abnormalities of Alzheimer's Disease in a Segmental Trisomy 16 Mouse Model of Down Syndrome
}

\author{
Anne M. Cataldo, ${ }^{1,2,3}$ Suzana Petanceska, ${ }^{3}$ Corrinne M. Peterhoff, ${ }^{3}$ Nicole B. Terio, ${ }^{3}$ Charles J. Epstein, ${ }^{4}$ Angela Villar, \\ Elaine J. Carlson, ${ }^{5}$ Matthias Staufenbiel, ${ }^{6}$ and Ralph A. Nixon ${ }^{3,7}$ \\ ${ }^{1}$ Mailman Research Center, McLean Hospital, Belmont, Massachusetts 02478, ${ }^{2}$ Department of Psychiatry, Harvard Medical School, Boston, Massachusetts \\ 02115, ${ }^{3}$ Center for Dementia Research, Nathan Kline Institute, Orangeburg, New York 10962, ${ }^{4}$ Department of Pediatrics, University of California, San \\ Francisco, San Francisco, California 94143, ${ }^{5}$ Genomics Core Facility, University of California, San Francisco, San Francisco, California 94143, ${ }^{6}$ Novartis \\ Institute of Biomedical Research, Nervous System, CH-4002 Basel, Switzerland, and 7Departments of Psychiatry and Cell Biology, New York University \\ School of Medicine, New York, New York 10016
}

\begin{abstract}
Altered neuronal endocytosis is the earliest known pathology in sporadic Alzheimer's disease (AD) and Down syndrome (DS) brain and has been linked to increased A $\beta$ production. Here, we show that a genetic model of DS (trisomy 21 ), the segmental trisomy 16 mouse Ts65Dn, develops enlarged neuronal early endosomes, increased immunoreactivity for markers of endosome fusion (rab5, early endosomal antigen 1, and rabaptin5), and endosome recycling (rab4) similar to those in AD and DS individuals. These abnormalities are most prominent in neurons of the basal forebrain, which later develop aging-related atrophy and degenerative changes, as in AD and DS. We also show that $A p p$, one of the triplicated genes in Ts65Dn mice and human DS, is critical to the development of these endocytic abnormalities. Selectively deleting one copy of $A p p$ or a small portion of the chromosome 16 segment containing App from Ts65Dn mice eliminated the endosomal phenotype. Overexpressing $A p p$ at high levels in mice did not alter early endosomes, implying that one or more additional genes on the triplicated segment of chromosome 16 are also required for the Ts65Dn endosomal phenotype. These results identify an essential role for App gene triplication in causing AD-related endosomal abnormalities and further establish the pathogenic significance of endosomal dysfunction in AD.
\end{abstract}

Key words: endosomes; endocytic pathway; Ts65Dn; Ts1Cje; Down syndrome; Alzheimer's disease; amyloid precursor protein; basal forebrain neurons

\section{Introduction}

Early endosomes of the endocytic pathway are the first intracellular, protease-rich sites involved in the internalization, recycling, and catabolic modulation of various macromolecules required for the normal maintenance of cells. The importance of the endocytic pathway to Alzheimer's disease (AD) pathogenesis is amply evidenced by its critical role in the processing and function of various proteins relevant to $\mathrm{AD}$, including amyloid precursor protein (APP), amyloid $\beta$ (A $\beta$ ) peptide, apolipoprotein $\mathrm{E}$ (ApoE), low-density lipoprotein, and low-density lipoprotein receptor-related protein (LRP), and by the localization of APP secretases or their activities, at least in part, within endosomes (Vassar et al., 1999; Huse et al., 2000).

Early endosomal alterations are the earliest known pathology in sporadic $\mathrm{AD}(\mathrm{SAD})$ and Down syndrome (DS), appearing in DS before birth, and in SAD, developing before $\beta$-amyloid is

Received April 7, 2003; revised April 7, 2003; accepted May 14, 2003.

This work was supported by National Institutes of Health Grants AG17617 and HD31498.

Correspondence should be addressed to Dr. Anne M. Cataldo, Mailman Research Center, McLean Hospital, 115 Mill Street, Belmont, MA 02472. E-mail: acataldo@mclean.harvard.edu.

Copyright $\odot 2003$ Society for Neuroscience $\quad$ 0270-6474/03/236788-05\$15.00/0 deposited and as soluble $\mathrm{A} \beta$ peptide levels first rise. Endosomal abnormalities, which are influenced by ApoE genotype (Cataldo et al., 1997), do not develop in familial AD caused by presenilin mutations (Cataldo et al., 2001), underscoring that they are AD subtype-specific and are also not a response to amyloid deposition. Similar endosomal abnormalities, when modeled in cells, are associated with increased $A \beta$ production (Grbovic et al., 2002). Endosomal alterations in AD and DS develop in otherwise normal-appearing neurons of regions that become the most severely affected in the disease (Cataldo et al., 1997, 2000a), including hippocampus, neocortex, and basal forebrain. In light of the suspected pathogenic importance of endosomal pathology in $\mathrm{AD}$, an animal model that reproduces these cellular alterations would be valuable.

Two segmental trisomy mouse models of DS, Ts65Dn (Davisson et al., 1990), and Ts1Cje (Sago et al., 1998) mice, survive to adulthood and exhibit a number of the morphological, biochemical, and transcriptional changes seen in the human disease (Davisson et al., 1990; Reeves et al., 1995; Holtzman et al., 1996). These animals possess three copies of the segment of mouse chromosome 16 (MMU16) orthologous to the critical region of human chromosome 21 (HSA21) thought to be responsible for the 
phenotype of DS. Ts65Dn mice with a segmental trisomy extending from $A p p$ to $M x 1$ exhibit behavioral and cognitive abnormalities not unlike some of the abnormalities seen in individuals with DS (Reeves et al., 1995). Ts65Dn mice also exhibit age-related atrophy and neurodegeneration of basal forebrain cholinergic neurons (BFCNs) and extensive astrocytic hypertrophy resembling these aspects of the neuropathology in AD and DS (Holtzman et al., 1996; Cooper et al., 2001). Unlike what occurs in human disorders, however, Ts65Dn mice do not deposit $\beta$-amyloid. Ts1Cje mice are genetically similar to Ts65Dn, except that the region from App to Sod1 is not triplicated. These mice do not develop BFCN atrophy and degeneration, and they have less severe learning and behavioral deficits than Ts65Dn mice (Sago et al., 1998).

In the present study, we established that Ts65Dn mice develop aging-related endosomal enlargement and altered expression and distribution of early endosome markers, which strongly resemble the neuronal endosomal pathology in SAD and DS. In addition, we investigated the role of App in the development of endosomal pathology using two additional types of trisomic mice, Ts1Cje and Ts65Dn-App $p^{+/+-}$, both of which possess only two copies of the App gene. These mice were also compared with transgenic mice expressing high levels of human mutated APP670/671 (Swedish mutation) or APP670/671 plus APP717 (London mutation). We demonstrate here that endosomal pathology in Ts65Dn mice is dependent on triplication of the App gene and that the ability of APP to alter endosome function requires the participation of one or more genes within a small trisomic region of MMU16. The influence on neuronal endocytic function of $A p p$ and $A p o E$, two genes that modify risk for $\mathrm{AD}$, provides strong support for the view that the very early-appearing abnormalities of endosomes in $\mathrm{AD}$ and DS have pathogenic significance.

\section{Materials and Methods}

Mice. Three segmental trisomy mice were used in this study. All mice were age-matched and housed with $2 \mathrm{~N}$ (diploid) littermates of the same sex. Ts65Dn mice $(n=6)$, which carry an extra copy of the distal end of MMU16 proximal to the App gene to Mxl (Davisson et al., 1990), were maintained on a $\mathrm{B} 6 \mathrm{C} 3 \mathrm{H}$ background and identified by karyotyping. Ts1Cje mice $(n=4)$, which are trisomic for the segment of MMU16 spanning from Sod1 to Mxl, were identified by neo-PCR and fluorescence in situ hybridization (Sago et al., 1998). App-mutant mice $\left(A p P^{-1-}\right)$ were generated and maintained on a C57BL/6J background (Zheng et al., 1995) and mated with Ts65Dn mice to generate Ts65Dn$A p p^{+/+1-}$ mice expressing two copies of the murine App gene $(n=6)$. Transgenic mouse lines that express the Swedish double mutation of APP (K670M/N671L) $(n=6)$ or the Swedish mutation plus the London mutation of human APP751 (V717I) were generated using transgenic constructs that contain human or murine Thy- 1 expression cassettes and human APP751 cDNAs, and mutant APP expression was confirmed as described previously (Sturchler-Pierrat et al., 1997). The presence of extracellular $\beta$-amyloid deposition was identified using thioflavin S. Transgenic mice carrying both mutant human APP and presenilin $1(n=6)$ transgenes were generated as described previously (Holcomb et al., 1998), and animals of both sexes ranging in age from 6 to 12 months were used in this study. The presence of transgene mRNAs was confirmed by PCR, and the presence of AD-like neuropathology was verified using thioflavin S histofluorescence.

Tissue. Trisomic, transgenic, and normal $2 \mathrm{~N}$ controls were fixed by transcardiac perfusion with aldehydes. Vibratome sections 30-40 $\mu \mathrm{m}$ thick that included regions of the medial septal nucleus (MSN), nucleus basalis magnocellularis (NBM), hippocampus, neocortex, basal ganglia, and cerebellum were collected from each animal.

Antibodies and immunocytochemistry. Immunocytochemical studies were performed as described previously (Cataldo et al., 1997, 2000a) using commercial antibodies to human rab5, rab4, and rabaptin5 and a

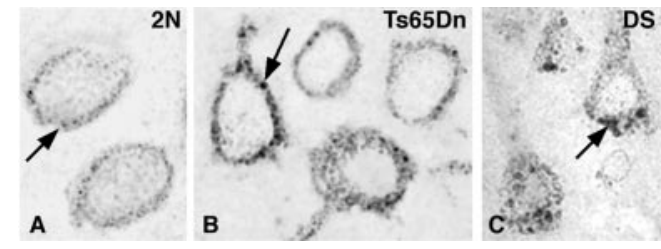

Figure 1. Early endosomal enlargement in neurons from Ts65Dn mice. Representative neurons from the brain of a 2-month-old Ts65Dn mouse $(B)$ labeled with rab5, showing the presence of enlarged early endosomes (arrow) as seen in DS ( $C$, arrow) and AD brain. In contrast to trisomic mice, neurons from age-matched $2 \mathrm{~N}$ control mice exhibit the typical small uniformly sized rab5-positive endosomes ( $A$, arrow).

purified polyclonal antibody raised against anti-early endosomal antigen 1 (EEA1) (Dr. S. Corvera, University of Massachusetts Medical School, Worcester, MA).

Western blot analysis. Brain hemispheres were homogenized in diethylamine (DEA)/50 mM NaCl at 1:10 w/v ratio and neutralized to $\mathrm{pH}$ 8.0. The pellets of the DEA extracts were solubilized in a $2 \%$ SDS-PBS mixture of protease inhibitors, sonicated, and boiled. Equal amounts of protein were sized by SDS-PAGE, and membranes were immunoblotted using anti-human rab4 (Cataldo et al., 2000a).

\section{Results}

\section{AD-like early endosomal abnormalities in neurons of Ts65Dn mice}

To investigate the presence of endosome alterations in the Ts65Dn mouse, we examined brain tissue from trisomic and $2 \mathrm{~N}$ control mice by immunocytochemistry with rab5, a specific marker for early endosomes. Neurons from Ts65Dn mice displayed rab5-positive early endosomes of abnormally large sizes (Fig. 1), which were similar morphologically to those seen in human DS brain as early as 2 months of age. In young Ts65Dn mice, these enlarged endosomes were prominent in a majority of the neurons in the MSN of the basal forebrain. Early endosomes in the same neuronal populations appeared as the typical, small, and uniform vesicular compartments in $2 \mathrm{~N}$ littermate control mice (Fig. 1).

To assess the functional significance of the endosomal enlargement in Ts65Dn mice, we examined the cellular expression of two known markers of endocytic function: rabaptin5 and EEA1. These proteins are recruited to early endosomal membranes through interactions with the GTP-active form of rab5 and modulate early endosomal docking and fusion (Stenmark et al., 1995; Gournier et al., 1998). Using antibodies to rabaptin5 and EEA1, we found that the pattern of rabaptin 5 and EEA1 in neurons from Ts65Dn mice resembled that of rab5, confirming the identity of the enlarged neuronal vacuolar profiles as early endosomes (Fig. 2) and implying that functionally, endosomal uptake and fusion were increased. As expected, the levels of rabaptin 5 and EEA1 immunoreactivities were higher in Ts65Dn mice than in age-matched littermate controls (Fig. 2).

Because the GTPase rab4 plays a functional role complementary to rab5 in directing the recycling of internalized membrane back to the cell surface (van der Sluijs et al., 1992), we also determined the levels and distribution of rab4 immunoreactivity in the MSN, NBM, neocortex and hippocampus from Ts65Dn and control mice. Rab4 immunoreactivity was principally associated with small vesicular profiles consistent with the size and location of recycling vesicles. We found a qualitative increase of rab4 immunolabeling in Ts65Dn mice, which was most prominent in neurons of the septohippocampal system and neocortex of Ts65Dn mice compared with age-matched $2 \mathrm{~N}$ controls (Fig. 2). This in- 


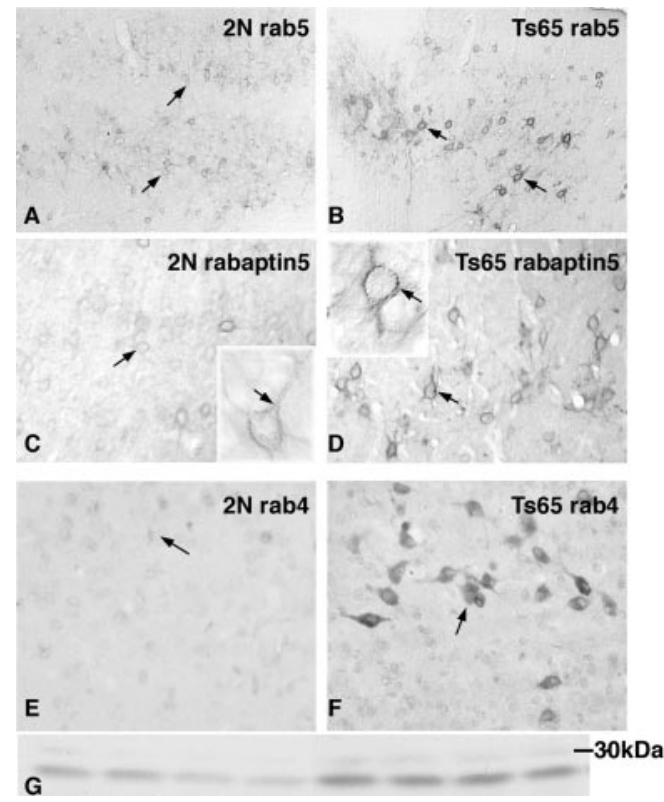

Figure 2. Endocytic uptake and recycling are increased in neurons of Ts65Dn mice. Neurons of the cingulate cortex of a 6-month-old Ts65Dn mouse labeled with rab5 ( $B$ ) and rabaptin5 (D) show increased immunoreactivity (arrows) and enlarged endosomes ( $D$, inset, arrow) compared with an age-matched $2 \mathrm{~N}$ control mouse $(A, C, C$, inset, arrows). Serial adjacent sections immunolabeled with rab4 show increased immunoreactivity $(F$, arrow) in neurons of trisomic mice compared with controls ( $E$, arrow), which is consistent with increased endosomal reflux to the plasma membrane. Western blot analysis ( $G$ ) of whole-brain homogenates (50 $\mu \mathrm{g}$ per lane) prepared from $2 \mathrm{~N}$ control mice ( $n=4$; lanes $1-4)$ and Ts65Dn mice $(n=4$; lanes 5-8) confirm the immunocytochemical findings and revealed an increase in rab4-immunoreactive protein $\left(M_{r} \sim 25-28\right)$ in the Ts65Dn mouse brains.

crease was confirmed by Western blot analysis, which revealed a 2.5 -fold increase in rab4 levels $(p<0.0006 ; n=4)$.

Given the importance of aging as a risk factor for $\mathrm{AD}$, we evaluated the effects of aging on the neuronal endocytic pathway by examining Ts65Dn mice and $2 \mathrm{~N}$ littermate controls at 6,12 , and 18 months of age. Immunocytochemistry showed that by 6 months of age, swollen, rab5-positive endosomes were present in most basal forebrain neurons of the MSN as well as the NBM. Endosomal enlargement was detected in fewer neurons within the amygdala, cingulate cortex, and hippocampus from Ts65Dn mice. The numbers of neurons in these regions containing abnormally large endosomes increased in Ts65Dn mice 12 months of age and older, although the magnitude of enlargement did not appear to differ from that seen in the young, 2-month-old mice. Not all neuronal populations in the Ts65Dn mice exhibited enlarged endosomes; neurons in the caudate nucleus and putamen (Fig. 3) or in cerebellar Purkinje cells displayed normal-sized early endosomes.

\section{App gene dosage modulates early endosomal morphology in Ts65Dn mice}

Because of the importance of the APP gene in AD pathogenesis, we investigated whether changes of the endocytic pathway detected in Ts65Dn were dependent on App gene dosage. We compared brain tissue from Ts65Dn mice with that from two different trisomic mouse strains that carry the normal two copies of the App gene. The first, the Ts1Cje mouse, lacks the trisomic segment of Ts65Dn from App to Sod1 (a segment containing $\sim 28$ genes). The second strain, a Ts65Dn-App ${ }^{+/+/-}$knock-out mouse generated by crossing Ts65Dn with an App knock-out mouse, is a segmental trisomy with two copies of the App gene. Rab5 immu-

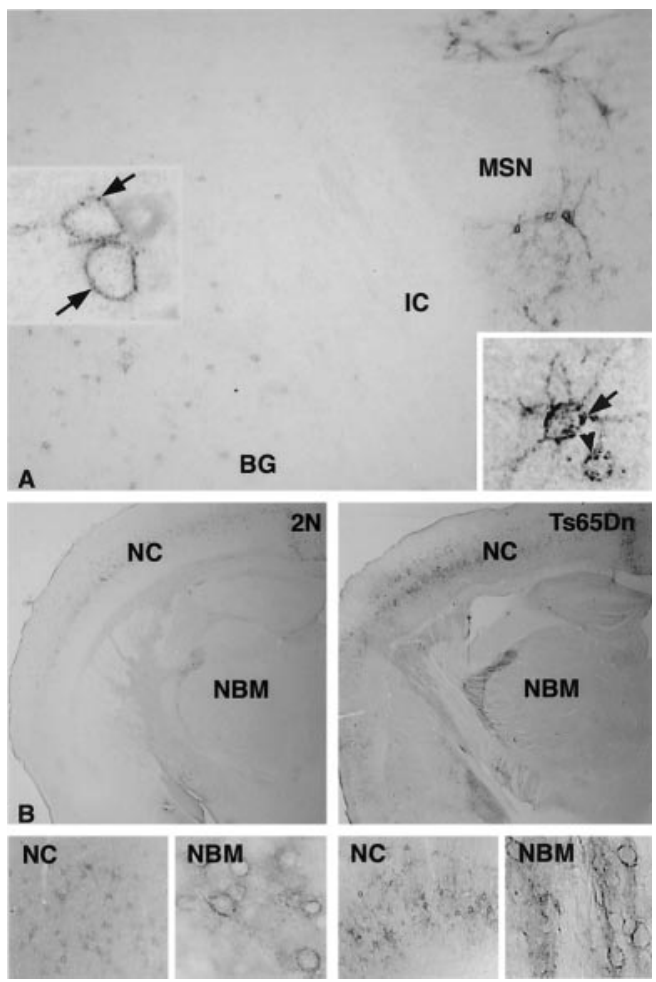

Figure 3. Endocytic abnormalities target neurons of the septohippocampal system. $A$, In tissue sections from 2-month-old Ts65Dn mice, endocytic abnormalities are most prominent in neurons of the medial septal nucleus (MSN). Increased levels of rab5 immunoreactivity and atypically large rab5-positive endosomes (right inset, arrows) are detected in most neurons in this region, which is composed primarily of cholinergic neurons that undergo age-related neurodegeneration in Ts65Dn mice. In neurons of the basal ganglia (BG), in contrast, rab5 $\mathrm{immu}$ nolabeling in neurons is associated with the typical small early endosomal profiles located in close proximity to the cell surface (left inset, arrows). IC, Internal capsule. B, Age-related endosomal alterations represented by enlarged rab5-positive endosomes and elevated levels of rab5 immunolabeling are apparent in neurons of the MSN as well as other regions of the septohippocampal system, including related populations in the neocortex and hippocampus and the NBM.

nocytochemistry showed that neurons of both of these mouse strains displayed early endosomes of normal size in the MSN, NBM, neocortex, and hippocampus in numbers qualitatively similar to that seen in neurons of $2 \mathrm{~N}$ brains (Fig. 4). In addition, both the Ts65Dn-App $p^{+/+/-}$and Ts1Cje mice expressed rab4 immunoreactivity at levels similar to those in neurons from agematched littermate controls.

To evaluate further the effects of App gene dosage on the promotion of endocytic disturbances, we examined brain tissue from transgenic mice overexpressing the Swedish mutation of human APP751 (APP670/671) alone or in combination with the London mutation of APP751 (APP670/671/717). A twofold overexpression of the combined Swedish and London mutations of APP and a sevenfold overexpression of the Swedish mutation of APP in these transgenic mice have been demonstrated previously (Sturchler-Pierrat et al., 1997). Basal forebrain neurons from both of these lines of App transgenic mice had normal-appearing vesicular rab5-positive early endosomes (Fig. 4) and patterns of immunolabeling for the other endosomal markers EEA1, rab4, and rabaptin5, similar to those in $2 \mathrm{~N}$ controls.

\section{Discussion}

We identified previously morphological abnormalities in $\mathrm{AD}$ and DS brain consistent with increased endocytic pathway activation, 


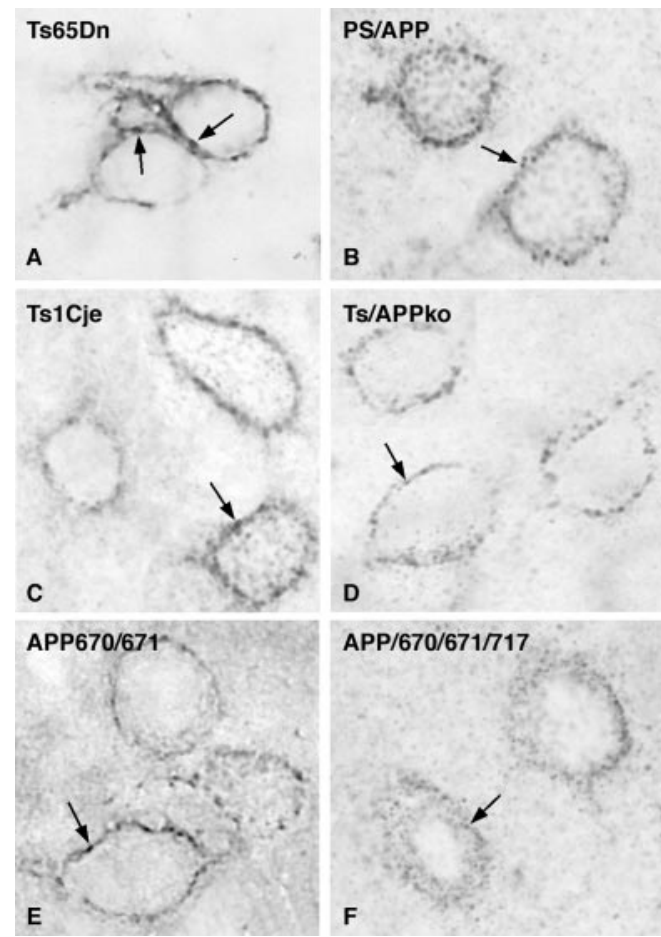

Figure 4. App triplication promotes endosomal abnormalities in Ts65Dn mice. Rab5positive early endosomes in representative basal forebrain neurons from a Ts65Dn mouse with three copies of the App gene $(A)$ are abnormally large (arrow) compared with those in control $2 \mathrm{~N}$ mice with two copies of App (Fig. 1). Neurons from the same neuronal populations in presenilin (PS)-APP transgenic mice $(B)$ and from two strains of trisomic mice with two copies of $A p p$, Ts1Cje ( $C$, and Ts65Dn-App ${ }^{+/+/-}(D)$ do not exhibit endosomal abnormalities but, like $2 \mathrm{~N}$ control mice, contained early endosomes of normal size (arrows). Mice transgenic for mutant forms of human $\operatorname{APP}(E, F)$ that express two- to sevenfold higher protein levels also show endosomes of normal size implying that App dosage alone does not promote the endocytic response.

including increased volumes of early endosomes, a well established morphological event associated with increased endocytosis (Bucci et al., 1992), and elevated expression of proteins that regulate endocytosis (rab5, rabaptin5, EEA1) and recycling (rab4) (Cataldo et al., 1997, 2000a). We also showed that endosomal pathology in DS occurs as early as 28 weeks gestation, decades before $\beta$-amyloid is deposited in significant amounts (Cataldo et al., 2000a). In AD, the appearance of abnormal endosomes coincides with rises in soluble $A \beta$ levels and the detection of $A \beta$ immunoreactivity intraneuronally (Cataldo et al., 2000b). In this study, we observed that Ts65Dn mice also develop aging-related enlargement of rab5-positive endosomes, which immunolabel more strongly with antibodies to two other effectors of endocytosis, rabaptin5 and EEA1, consistent with the conclusion that endocytic uptake and subsequent endosomal docking and fusion are increased in these neurons. A compensatory rise in levels of the GTPase rab4, which stimulates endosome recycling to the plasmalemma, was also detected in the same neuronal groups. These similarities to DS and AD establish Ts65Dn mice as a useful model to study the nature and pathophysiological consequences of endosome dysfunction, the earliest known cellular pathology in $\mathrm{AD}$ (Cataldo et al., 1997, 2000a, 2001).

We have shown that the development of early endosomal abnormalities in Ts65Dn mice is dependent on App gene triplication. Ts1Cje and TsDn65-App $p^{+/+/-}$mice, which have two copies of $A p p$, showed normal endosomal morphology in the basal forebrain in contrast to Ts65Dn mice. These same populations of basal forebrain neurons typically develop less severe atrophy in the Ts1Cje (Sago et al., 1998) and Ts65Dn-App ${ }^{+/+/-}$mice (our unpublished observation) or older ages compared with that occurring in age-matched Ts65Dn mice. These findings are consistent with observations that endosomal abnormalities in DS develop in some neurons before birth, which links this phenomenon with dosage of particular genes on the triplicated segment of chromosome 21. It is relevant, in this regard, that another major genetic risk factor for $\mathrm{AD}$, the ApoE4 allele, also accelerates the onset of endosome pathology in $\mathrm{AD}$ (Corder et al., 1993). ApoE, its receptor on neurons (LRP), and one of its ligands, cholesterol, which are trafficked through endosomes at increased rates during neuronal injury, have each been implicated in $\mathrm{AD}$ pathogenesis. Given the importance of the App gene to $\mathrm{AD}$ pathogenesis and the high disease specificity of neuronal endocytic pathology, our observation that App triplication is required for the endosomal phenotype in the mouse strongly supports the view that endocytic pathway dysfunction is a key pathogenic event in $\mathrm{AD}$.

Although increased App gene dosage is necessary for endosome pathology to develop in Ts65Dn mice, it is not sufficient, because overexpressing APP alone did not cause endosome pathology or atrophy of basal forebrain neurons. These findings imply that one or more genes in the trisomic portion of MMU16 are also necessary. Of the $\sim 150$ genes on MMU16 that are syntenic with HSA21, 60\% are expressed in brain, and most of these are present in postmitotic cells. Among these genes are several with potential relevance to $\mathrm{AD}$. Oxidative stress is a possible contributory factor in DS and AD pathogenesis, and decreased viability of fetal DS neurons in culture has been linked to increased reactive oxygen species (Busciglio and Yankner, 1995). In this regard, Sod1 triplication may be contributory on the basis of evidence that increased Sod1 expression in DS occurs in the absence of a compensatory increase in catalase or glutathione peroxidase leading to an accumulation of hydrogen peroxide or free hydroxyl radical, both of which are cytotoxic. $\beta$-site APP cleaving enzyme (BACE)-2 is a transmembrane aspartic protease that is related to the major $\beta$-secretase $\mathrm{BACE}-1$, an $\mathrm{AD}$-relevant protein located in endosomes. Immunocytochemical studies of brain tissue from DS subjects have shown a direct correlation between the appearance of elevated levels of BACE-2 in brain and the presence of AD-like neuropathology (Motonaga et al., 2002). $S 100 \beta$ is another triplicated gene on HSA21 that encodes a small acidic calcium-binding protein synthesized and released by astrocytes (Allore et al., 1988). S100 $\beta$ expression is increased as early as 17 weeks gestation in DS brain (Griffin et al., 1989, 1998), and it is believed to contribute to APP overexpression (Griffin et al., 1989, 1998), dendritic abnormalities, dystrophic neurites, and apoptosis (Sheng et al., 1997). Cholinergic neurons have been shown to be particularly vulnerable to the cytotoxic effects of inflammatory cytokines, including interleukins and $S 100 \beta$ by astrocytes (Wenk and Willard, 1998). Finally, the ApoE gene, which is associated with increased risk of AD (Corder et al., 1993), is not located on the triplicated segment of MMU16, but it is overexpressed to higher levels in Ts65Dn mice than is predicted on the basis of gene dosage (Holtzman et al., 1996). It is possible that genes not on the trisomic segment could interact with App to promote endocytic dysfunction in Ts65Dn mouse, as ApoE4 does in AD.

Our findings show that Ts65Dn mice develop neuronal endosome abnormalities strongly resembling the highly $\mathrm{AD}$-specific endocytic alterations that develop at the earliest stages of $\mathrm{AD}$. Moreover, similar endosome alterations modeled in cells by overexpressing rab5 increase $\mathrm{A} \beta$ peptide generation (Grbovic et 
al., 2002), which may account for the disproportionate elevation of $\mathrm{A} \beta$ levels compared with APP expression in Ts65Dn mice. As seen in neurons from infants and fetuses with trisomy 21 (Cataldo et al., 2000b), we also found intraneuronal $\mathrm{A} \beta$ within rab5-positive vesicular compartments of neurons from Ts65Dn mice. Although Ts65Dn mice (even at 24 months of age) do not exhibit extracellular $\beta$-amyloid-containing plaques (Holtzman et al., 1996) or neurofibrillary tangles, this late-stage AD pathology develops in mice only when human APP or tau in mutant form is expressed at high levels. This suggests differential effects of factors such as aging or diet among species on disease phenotype in the advanced stages. Despite the lack of development of late-stage pathology in Ts65Dn mice, the association between endosomal disturbances and basal forebrain neuronal atrophy and its close relationship to App gene dosage support the use of Ts65Dn mice as a valuable model in which to investigate the key early events of $\mathrm{AD}$ pathogenesis.

\section{References}

Allore R, O'Hanlon D, Price R, Neilson K, Willard HF, Cox DR, Marks A, Dunn RJ (1988) Gene encoding the beta subunit of S100 protein is on chromosome 21: implications for Down syndrome. Science 239:1311-1313.

Bucci C, Parton RG, Mather IH, Stunnenberg H, Simons K, Hoflack B, Zerial M (1992) The small GTPase rab5 functions as a regulatory factor in the early endocytic pathway. Cell 70:715-728.

Busciglio J, Yankner BA (1995) Apoptosis and increased generation of reactive oxygen species in Down's syndrome neurons in vitro. Nature 378:776-779.

Cataldo AM, Barnett JL, Pieroni C, Nixon RA (1997) Increased neuronal endocytosis and protease delivery to early endosomes in sporadic Alzheimer's disease: neuropathologic evidence for a mechanism of increased $\beta$-amyloidogenesis. J Neurosci 17:6142-6151.

Cataldo AM, Peterhoff CM, Troncoso JC, Gomez-Isla T, Hyman BT, Nixon RA (2000a) Endocytic pathway abnormalities precede amyloid beta deposition in sporadic Alzheimer's disease and Down syndrome: differential effects of APOE genotype and presenilin mutations. Am J Pathol 157:277-286

Cataldo AM, Nixon RA, Troncoso J, Durham R, Buxbaum J, Epstein C, Carlson E, Peterhoff C (2000b) Endocytic alterations in human preclinical Alzheimer's disease and a trisomic mouse model of Down syndrome: implications for $\beta$-amyloidogenesis. Neurobiol Aging 21:293.

Cataldo AM, Rebeck GW, Ghetti B, Hulette C, Lippa C, van Broeckhoven C, van Duijn C, Cras P, Bogdanovic N, Bird T, Peterhoff CM, Nixon RA (2001) Endocytic disturbances distinguish among subtypes of Alzheimer's disease and related disorders. Ann Neurol 50:661-665.

Cooper J, Salehi A, Delcroix JD, Howe CL, Belichenko PV, Chua-Couzens J, Kilbridge JK, Carlson EJ, Epstein CJ, Mobley WC (2001) Failed retrograde transport of NGF in a mouse model of Down's syndrome: reversal of cholinergic neurodegenerative phenotypes following NGF infusion. Proc Natl Acad Sci USA 98:10439-10444.

Corder EH, Saunders AM, Strittmatter WJ, Schmechel DE, Gaskell PC (1993) Gene dose of apolipoprotein E type 4 allele and the risk of Alzheimer's disease in late onset families. Science 261:921-923.

Davisson MT, Schmidt C, Akeson EC (1990) Segmental trisomy of murine chromosome 16: new model system for studying Down syndrome. Prog Clin Biol Res 360:263-280.

Gournier H, Stenmark H, Rybin V, Lippe R, Zerial M (1998) Two distinct effectors of the small GTPase Rab5 cooperate in endocytic membrane fusion. EMBO J 17:1930-1940.
Grbovic OM, Mathews PM, Schmidt SD, Nixon RA, Cataldo AM (2002) Rab5 Overexpressionin a cell model of AD-related endocytic abnormalities influences processing of $\beta$ APP. Neurobiol Aging 23:S13:50.

Griffin WST, Stanley LC, Ling C, White L, MacLeod V, Perrot LJ, White CL, Araoz C (1989) Brain interleukin 1 and S-100 immunoreactivity are elevated in Down syndrome and Alzheimer disease. Proc Natl Acad Sci USA 86:7611-7615.

Griffin WST, Sheng JG, McKenzie LE, Royston MC, Gentleman SM, Brumback RA, Cork LC, Del Bigio MR, Roberts GW, Mrak RE (1998) Lifelong overexpression of S100beta in Down's syndrome: implications for Alzheimer pathogenesis. Neurobiol Aging 19:401-405.

Holcomb L, Gordon M, McGowan E, Yu X, Benkovic S, Jantzen P, Wright K, Saad I, Mueller R, Morgan D, Sanders S, Zehr C, O’Campo K, Hardy J Prada CM, Eckman C, Younkin S, Hsaio K, Duff K (1998) Accelerated Alzheimer-type phenotype in transgenic mice carrying both mutant amyloid precursor protein and presenilin 1 transgenes. Nat Med 1:97-100.

Holtzman DM, Santucci D, Kilbridge J, Chua-Couzons J, Fontana DJ, Daniels SE, Johnson RM, Chen K, Sung Y, Carlson E, Alleva E, Epstein CJ, Mobley WC (1996) Developmental abnormalities and age-related neurodegeneration in a mouse model of Down syndrome. Proc Natl Acad Sci USA 93:13333-13338.

Huse JT, Pijak DS, Leslie GJ, Lee VM-Y, Doms RW (2000) Maturation and endosomal targeting of beta-site amyloid precursor protein-cleaving enzyme. The Alzheimer's disease beta-secretase. J Biol Chem 275:33729-33737.

Motonaga K, Itoh M, Becker LE, Goto Y, Takashima S (2002) Elevated expression of beta-site amyloid precursor protein cleaving enzyme 2 in brains of patients with Down syndrome. Neurosci Lett 326:64-66.

Reeves RH, Irving N, Moran T, Wohn A, Kitt C, Sisodia SS, Schmidt C, Bronson R, Davisson M (1995) A mouse model for Down syndrome exhibits learning and behaviour deficits. Nat Genet 11:177-184.

Sago H, Carlson EJ, Smith DJ, Kilbridge J, Rubin EM, Mobley WC, Epstein CJ, Huang TT (1998) Ts1Cje, a partial trisomy 16 mouse model for Down syndrome, exhibits learning and behavioral abnormalities. Proc Natl Acad Sci USA 95:6256-6261.

Sheng JG, Mrak RE, Griffin WST (1997) Glial-neuronal interactions in Alzheimer disease: progressive association of IL-1alpha+ microglia and S100beta + astrocytes with neurofibrillary tangle stages. J Neuropathol Exp Neurol 56:285-290.

Stenmark H, Vitale G, Ullrich O, Zerial M (1995) Rabaptin-5 is a direct effector of the small GTPase Rab5 in endocytic membrane fusion. Cell 83:423-432.

Sturchler-Pierrat C, Abramowski D, Duke M, Wiederhold KH, Mistl C, Rothacher S, Ledermann B, Burki K, Frey P, Paganetti PA, Waridel C, Calhoun ME, Jucker M, Probst A, Staufenbiel M, Sommer B (1997) Two amyloid precursor protein transgenic mouse models with Alzheimer disease-like pathology. Proc Natl Acad Sci USA 94:13287-13292.

van der Sluijs P, Hull M, Webster P, Male P, Goud B, Mellman I (1992) The small GTP-binding protein rab4 controls an early sorting event on the endocytic pathway. Cell 70:729-740.

Vassar R, Bennett BD, Babu-Khan S, Mendiaz EA, Denis P, Teplow DB, Ross S, Amarante P, Loeloff R, Luo Y, Fisher S, Fuller J, Edenson S, Lile J, Jarosinski MA, Biere AL, Curran E, Burgess T, Louis JC, Collins F, et al. (1999) Beta-secretase cleavage of Alzheimer's amyloid precursor protein by the transmembrane aspartic protease BACE. Science 286:735-741.

Wenk GL, Willard LB (1998) The neural mechanisms underlying cholinergic cell death within the basal forebrain. Int J Dev Neurosci 16:729-735.

Zheng H, Jiang M, Trumbauer ME, Sirinathsinghji DJS, Hopkins R, Smith DW, Heavens RP, Dawson DW, Boyce S, Connor MW, Stevens KA, Slunt HH, Sisodia SS, Chen HY, Van der Ploeg LHT (1995) beta-Amyloid precursor protein-deficient mice show reactive gliosis and decreased locomotor activity. Cell 81:525-531. 